Takeshi Miyamoto - Naomi Kanazawa - Shigeo Kato

Masato Kawakami • Yoshito Inoue • Tomiko Kuhara

Takehiko Inoue $\cdot$ Kenzo Takeshita $\cdot$ Seiichi Tsujino

\title{
Diagnosis of Japanese patients with HHH syndrome by molecular genetic analysis: a common mutation, $\mathrm{R} 179 \mathrm{X}$
}

Received: January 18, 2001 / Accepted: January 31, 2001

\begin{abstract}
Patients with mitochondrial ornithine transporter deficiency (or HHH syndrome) present with various neurological symptoms, including mental retardation, spastic paraparesis with pyramidal signs, cerebellar ataxia, and episodic disturbance of consciousness or coma due to hyperammonemia. We previously described three novel mutations in the ORNT1 gene in Japanese patients with $\mathrm{HHH}$ syndrome. In this article, we report a new patient with HHH syndrome, a 52-year-old woman, who had the typical clinical features, except for an absence of mental retardation. When we screened this patient, as well as a previously described Japanese patient, for mutations in the ORNT1 gene, we found that both were homozygous for a nonsense mutation (R179X). Furthermore, reverse transcription (RT)-polymerase chain reaction (PCR) of fibroblast RNA from one patient showed exon 4 skipping, as had been observed in a previously reported patient with R179X. These results, together with the findings in our
\end{abstract}

T. Miyamoto $\cdot$ N. Kanazawa $\cdot$ S. Tsujino $(\bowtie)$

Department of Inherited Metabolic Disease, National Institute of

Neuroscience, NCNP, 4-1-1 Ogawahigashi, Kodaira, Tokyo 187-8502, Japan

Tel. +81-42-341-2711; Fax +81-42-346-1746

e-mail: tsujino@ncnp.go.jp

N. Kanazawa

Research Division of Biochemistry, Kitasato University School of Medicine, Kanagawa, Japan

S. Kato

Department of Internal Medicine, Shinfuji Hospital, Shizuoka, Japan

M. Kawakami

Department of Neurology, St. Marianna University School of

Medicine, Kanagawa, Japan

Y. Inoue $\cdot$ T. Kuhara

Biochemical Section, Division of Human Genetics, Medical Research

Institute, Kanazawa Medical University, Ishikawa, Japan

T. Inoue

The Department of Pediatrics, Western Shimane Medical and

Welfare Center for the Handicapped, Shimane, Japan

K. Takeshita

Division of Child Neurology, Institute of Neurological Sciences,

Faculty of Medicine, Tottori University, Yonago, Japan previous report, show that, in three of our five reported Japanese HHH patients (six of ten alleles), R179X is present, suggesting that this is a common mutation in Japanese patients with HHH syndrome.

Key words $\mathrm{HHH}$ syndrome $\cdot$ Ornithine transporter $\cdot$ Mitochondria $\cdot$ Mutation $\cdot$ ORNT1

\section{Introduction}

Mitochondrial ornithine transporter deficiency, or $\mathrm{HHH}$ syndrome (MIM 238970) is characterized by three biochemical abnormalities; hyperornithinemia, hyperammonemia, and homocitrullinuria (Camacho et al. 1999). Clinically, HHH syndrome presents with mental retardation, seizures, spastic paraparesis with pyramidal signs, cerebellar ataxia, and episodic disturbance of consciousness or coma due to hyperammonemia (Valle and Simell 1995). More than 50 patients have been reported since Shih et al. first reported this syndrome in 1969. We previously reported three novel mutations in the ORNT1 gene in Japanese patients with $\mathrm{HHH}$ syndrome (Tsujino et al. 2000).

In this article, we report a new patient, as well as the results of screening this patient and a previously reported Japanese patient for the three mutations reported by Tsujino et al. (2000), using polymerase chain reaction (PCR)-restriction fragment length polymorphism (RFLP) analysis and the amplification refractory mutation system (ARMS) method.

\section{Patients and methods}

Patient 1

Patient 1, a 52-year-old woman, had spastic gait and cerebellar signs such as dysmetria, dysdiadochokinesis, and scanning speech, since adolescence. There was no information about consanguinity. She had had spastic paraparesis 
and paroxysmal involvement of the upper extremities since the age of 27 years. At the age of 36 years, hyperammonemia was noted, and a protein-restricted diet and kanamycin were prescribed. At age 37, she complained of tension in her legs and had surgery to elongate the Achilles tendons in both legs. When she was diagnosed with $\mathrm{HHH}$ syndrome at age 52, the ornithine concentration in serum was elevated, at $373 \mathrm{nmol} / \mu \mathrm{l}$ (normal range, $36-110 \mathrm{nmol} /$ $\mu \mathrm{l}$ ), and homocitrullinuria was shown. Her blood ammonia level after meals was increased, at 126-404 $\mu \mathrm{g} / \mathrm{dl}$ (normal range, less than $80 \mu \mathrm{g} / \mathrm{dl})$.

\section{Patient 2}

Patient 2 was previously described elsewhere (Nakajima et al. 1988). Briefly, He was a 10 -year-old boy, with healthy Japanese parents who are first cousins. He was delivered at 38 weeks gestation, with a birth weight of $1850 \mathrm{~g}$. His developmental milestones were delayed in late infancy. At the age of 3 years, spastic paraplegia was noted. He always avoided the intake of meat, milk, and eggs. At the age of 10, he had an episode of lethargy and hyperammonemia. Neurological examination showed spastic diplegia, hyperkinetic behavior, severe mental retardation, and truncal ataxia. On examination, his fasting blood ammonia level was found to be $185-340 \mu \mathrm{g} / \mathrm{dl}$ (normal range, less than $80 \mu \mathrm{g} / \mathrm{dl}$ ), serum ornithine ranged between 185 and $514 \mu \mathrm{g} / \mathrm{dl}$ (normal range, $50-80 \mu \mathrm{g} / \mathrm{dl}$ ), and a large amount of homocitrulline was excreted in the urine.

\section{PCR amplification of genomic DNA and sequencing}

Genomic DNA was isolated from peripheral leukocytes or cultured skin fibroblasts of the two patients and a normal control. Intronic primers 1 (5'-tccttactagtgcttgatcag- $\left.3^{\prime}\right)$ and 2 (5'-ctgcttcaggcctgtcctaa-3') amplified a DNA fragment containing exon 4 by PCR as described (Tsujino et al. 2000). For sequencing, the PCR products were purified with a GeneElute Agarose Spin Column (Sigma, St Louis, MO, USA) or with a QIAquick PCR purification Kit (Qiagen, Hilden, Germany); direct sequencing was done with the Dye Terminator Cycle Sequencing FS Ready Reaction Kit (PE Biosystems, Foster City, CA, USA) with primers 1 and 2 and an ABI Prism 310 Genetic Analyzer (PE Biosystems), according to the manufacturer's instructions.

PCR-based detection of the three mutations

A PCR fragment with primers 1 and 2 was digested with $\operatorname{Taq}^{\alpha} \mathrm{I}$ (New England Biolabs, Beverly, MA, USA). In the presence of the nonsense mutation (R179X), the PCR product was not cleaved by the restriction endonuclease $T a q^{\alpha} \mathrm{I}$, whereas the wild-type PCR product was cleaved (Fig. 1A). Restriction digests were analyzed in $2 \%$ NuSieve (Rockland, ME, USA) agarose. Detection of the two other mutations (G27E or 228/229 ins AAC) was performed with PCR-RFLP or ARMS as described (Tsujino et al. 2000).
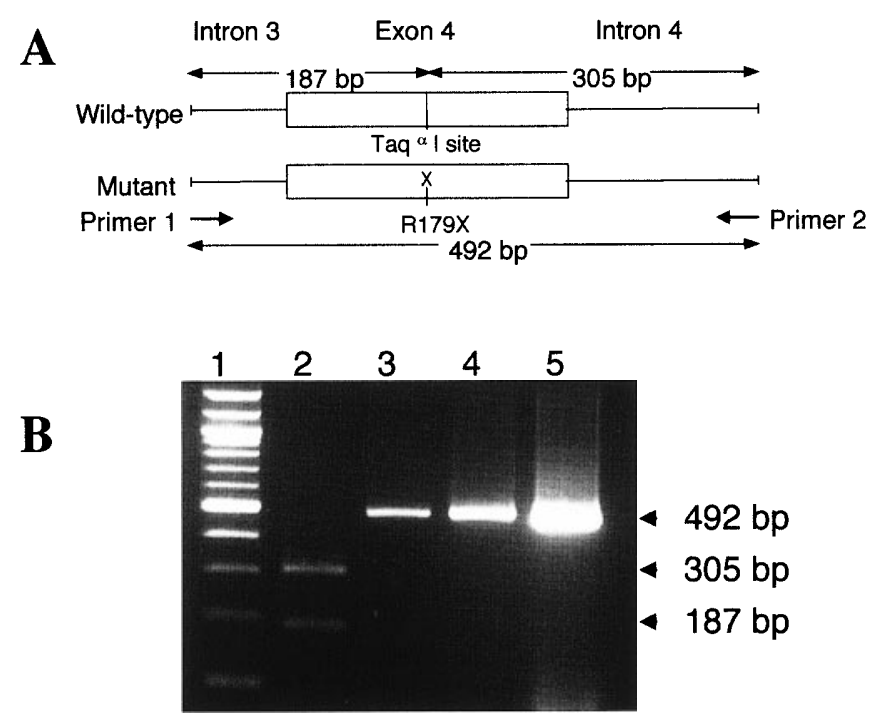

Fig. 1. A Schematic representation of the wild-type PCR fragment and the mutant PCR fragment. Boxes indicate exon 4. Base pair numbers indicate lengths of segments cleaved by $T a q^{\alpha} \mathrm{I}$. B Electrophoresis, on $2 \%$ NuSieve agarose, of PCR products containing R179X after digestion with $T a q^{\alpha}$ I. Lane 1, DNA size marker 100-bp ladder; lane 2, normal control; lane 3, positive control (a previously described patient with $\mathrm{R} 179 \mathrm{X})$; lane 4, patient 1; lane 5, patient 2

\section{Results and discussion}

Digestion of the PCR fragment containing exon 4 with $T a q^{\alpha}$ I revealed that both patients were homozygous for a C-to-T transition, resulting in a change of CGA to TGA at codon 179 (R179X)(Fig. 1B). This was confirmed by sequencing the PCR products. Neither patient had either of the other two mutations (G27E or 228/229 ins AAC). We also detected exon 4 skipping in the reverse transcription (RT)-PCR product of patient 2, similar to what we had found previously in another patient with R179X (Tsujino et al. 2000).

Camacho et al. (1999) reported two mutations and a microdeletion in 11 patients with $\mathrm{HHH}$ syndrome (Table 1). One patient was a genetic compound heterozygote. He had the $\mathrm{E} 180 \mathrm{~K}$ mutation on one allele, which he inherited from his father, who was Irish-American and was heterozygous for $\mathrm{E} 180 \mathrm{~K}$. On the other allele, he carried a microdeletion involving $O R N T 1$, which he inherited from his mother, who was Japanese. She did not have the E180K mutation, but was heterozygous for the microdeletion. The other 10 patients were all French-Canadian, 9 of whom were homozygous for the F188 deletion. The remaining patient was a compound heterozygote for the F188 deletion and an as yet unknown mutation. Shih and Ficicioglu (2000) also reported mutations in 11 patients with HHH syndrome. An A15E mutation was identified in a Palestinian infant and a CA insertion following the 96th nucleotide in a South American girl. The other 9 patients were French-Canadian, 7 of whom were homozygous and 2 of whom were heterozy- 
Table 1. Summary of mutations described in HHH syndrome

\begin{tabular}{llll}
\hline Mutation & Ethnic background & Comment & Reference \\
\hline F188 deletion & French-Canadian & $\begin{array}{c}\text { Common in French-Canadian patients } \\
(35 / 38 \text { alleles })^{\mathrm{a}}\end{array}$ & Camacho et al. (1999); Shih and Ficicioglu (2000) $^{\mathrm{a}}$ \\
E180K & Irish-American & & Camacho et al. (1999) \\
A15E & Palestinian & & Shih and Ficicioglu (2000) \\
96/97 insCA & South American & & Shih and Ficicioglu (2000) \\
Microdeletion & Japanese & Common in Japanese patients (6/10 alleles) & Camacho et al. (1999) \\
R179X & Japajino et al. (2000) \\
G27E & Japanese & & Tsujino et al. (2000) \\
228/229 insAAC & Japanese & & Tsujino et al. (2000) \\
\hline
\end{tabular}

${ }^{a}$ The number of alleles is the total of those in these two reports

gous for the F188 deletion (the second allele in these 2 patients was not identified).

The results in the present study, together with the findings in our previous report, show that, in three of our five reported Japanese HHH patients (six of ten alleles), R179X is present, suggesting that this is a common mutation in Japanese patients with $\mathrm{HHH}$ syndrome. The home towns of the three Japanese patients with R179X were Nima-gun (Shimane prefecture), Onomichi (Hiroshima prefecture), and Numazu (Shizuoka prefecture). While the towns of Nima-gun and Onomichi are comparatively close to each other, Numazu is far from these two towns. This suggests that the R179X allele may be widely distributed in Japan. Although it is believed that $\mathrm{HHH}$ syndrome is a relatively rare disorder, there may be additional Japanese patients who remain undiagnosed. We conclude that screening for R179X may be useful for the diagnosis of $\mathrm{HHH}$ syndrome in Japanese patients.

Acknowledgements We thank Dr. Yu-ichi Takusa and Dr. Seiji Yamaguchi (Shimane Medical University) for providing information on a patient, Dr. Toyokazu Saito (Kitasato University East Hospital) for his support, Ms. Kazuko Endate for technical assistance, and Dr. Sara Shanske (Columbia University) for reviewing the manuscript. This work was partially supported by a Grant-in-Aid for Scientific Research from the Japan Society for the Promotion of Science.

\section{References}

Camacho JA, Obie C, Biery B, Goodman BK, Hu CA, Almashanu S, Steel G, Casey R, Lambert M, Mitchell GA, Valle D (1999) Hyperornithinaemia-hyperammonaemia-homocitrullinuria syndrome is caused by mutations in a gene encoding a mitochondrial ornithine transporter. Nat Genet 22:151-158

Nakajima M, Ishii S, Mito T, Takeshita K, Takashima S, Takakura H, Inoue I, Saheki T, Akiyoshi H, Ichihara K (1988) Clinical, biochemical and ultrastructural study on the pathogenesis of hyperornithinemia-hyperammonemia-homocitrullinuria syndrome. Brain Dev 10:181-185

Shih V, Efron M, Moswe H (1969) Hyperornithinemia, hyperammonemia, and homocitrullinuria: A new disorder of amino acid metabolism associated with myoclonic seizures and mental retardation. Am J Dis Child 117:83-92

Shih V, Ficicioglu C (2000) Genotype and phenotype findings in the hyperornithinemia-hyperammonemia-homocitrullinuria (HHH) syndrome. J Inherit Metab Dis 23 (Suppl 1):72

Tsujino S, Kanazawa N, Ohashi T, Eto Y, Saito T, Kira J, Yamada T (2000) Three novel mutations (G27E, insAAC, R179X) in the ORNT1 gene of Japanese patients with hyperornithinemia, hyperammonemia, and homocitrullinuria syndrome. Ann Neurol 47:625-631

Valle D, Simell O (1995) The hyperornithinemia. In: Scriver C, Beaudet A, Sly W, Valle D (eds) The metabolic and molecular bases of inherited disease, 7th edn. McGraw Hill, New York, pp 1147-1185 